\title{
Abdominal-based adipocutaneous advancement flap for reconstructing inguinal defects with contraindications to standard reconstructive approaches: a simple and safe salvage reconstructive option
}

\author{
Clara Schaffer ${ }^{1}$, Daniel Haselbach ${ }^{1}$, Luigi Schiraldi ${ }^{1}$, Karl Sörelius ${ }^{2}$, Daniel F. Kalbermatten ${ }^{3}$, \\ Wassim Raffoul ${ }^{1}$, Pietro G. di Summa ${ }^{1}$ \\ ${ }^{1}$ Department of Plastic, Reconstructive and Hand Surgery, University Hospital of Lausanne (CHUV), Lausanne, Switzerland; ${ }^{2}$ Department \\ of Surgical Sciences, Section of Vascular Surgery, Uppsala University, Uppsala, Sweden; ${ }^{3}$ Department of Plastic, Reconstructive and Hand \\ Surgery, University Hospital of Basel, Basel, Switzerland
}

\begin{abstract}
Background Groin wounds occurring after vascular surgical site infection, oncologic resection, or occasionally orthopedic surgery and trauma may represent a surgical challenge. Reconstruction of these defects by the usual workhorse flaps may be contraindicated following previous surgery and in patients with lower limb lymphedema or extreme morbidity.

Methods This study included 15 consecutive patients presenting with inguinal wounds after vascular or general surgery that required debridement and soft tissue coverage. All cases had absolute or relative contraindications to conventional reconstructive techniques, including a compromised deep femoral artery network, limb lymphedema, scarring of potential flap harvesting sites, or poor overall condition. Abdominal adipocutaneous excess enabled the performance of adipocutaneous advancement flaps in an abdominoplasty-like fashion. Immediate and long-term outcomes were analyzed.

Results Soft tissue coverage was effective in all cases. Two patients required re-intervention due to flap-related complications (venous congestion and partial flap necrosis). All patients fully recovered over a mean \pm standard deviation follow-up of $2.4 \pm 1.5$ years.

Conclusions Abdominal flaps can be an effective and simple alternative technique for inguinal coverage with reproducible outcomes. In our experience, the main indications are a compromised deep femoral artery network and poor thigh tissue quality. Relative contraindications, such as previous open abdominal surgery, should be considered.
\end{abstract}

Keywords Abdomen / Groin / Infection / Reconstruction / Vascular surgery
Correspondence: Pietro G. di Summa Department of Plastic, Reconstructive and Hand Surgery, Centre Hospitalier Universitaire Vaudois (CHUV), Rue du Bugnon 46, Lausanne 1011,

Switzerland

Tel: +41-21-314-25-25

Fax: +41-21-314-25-30

E-mail: pietro.di-summa@chuv.ch

This article was presented at the 52nd Congress of Swiss Plastic Surgery (SGPRAC-SSCPRE) on September 9-10, 2016, in Lausanne, Switzerland.

The authors are grateful to Carol de Simio (Division of Surgical Research, University of Zürich [UZH], Zürich, Switzerland) for her illustrations, patience, and commitment. 


\section{INTRODUCTION}

Wounds in the groin area occurring after vascular surgical site infection (VSSI), oncologic resection, or occasionally orthopedic surgery and trauma may represent a surgical challenge [1]. VSSI alone has a mortality rate reaching $60 \%$, making effective soft tissue coverage a priority $[2,3]$. After failure of conservative treatment with local wound care and/or vacuum-assisted wound closure, groin wounds may require surgical management to cover major underlying structures, such as nerves and vessels, and to protect exposed prosthetic material. Common complications after reconstructive procedures include delayed healing, wound dehiscence, and persistence of the infection [4,5]. Standard reconstruction techniques use myocutaneous, fasciocutaneous, or muscular pedicle flaps, all of which commonly rely on the superficial femoral artery network, as with the sartorius muscle flap [6], or the deep femoral artery network. Indeed, the descending branch of the lateral circumflex femoral artery supplies the anterolateral thigh (ALT) flap [7], the rectus femoris muscle flap [8], and the vastus lateralis muscle flap [9]. The transverse and medial branches supply the tensor fascia lata flap and the gracilis muscle flap [10], respectively.

However, previous surgical procedures may limit the reconstructive options. In VSSI, the jeopardized proximal deep femoral artery network leads to the preferred use of rectus abdominis (RA) musculocutaneous or muscular flaps based on the supply from the deep inferior epigastric artery. The sartorius muscle flap, which is commonly used by vascular surgeons, represents a suboptimal solution due to its limited bulk [6], as well as its type IV vascularization; according to Mathes and Nahai [11], it relies on multiple minor pedicles that may be harmed by debridement. Scar tissue and lymphedema, which are potentially encountered after vascular access, VSSI, or orthopedic surgery, may pose issues at the harvest sites of commonly used flaps. Furthermore, severe comorbidities, such as cardiac failure or wasting syndrome, are contraindications for complex surgical procedures. These specific situations require a simple salvage procedure to promote healing by restoring soft tissue coverage of the groin area.

Anatomically, the abdominal wall is divided into three areas in terms of vascular supply, with anastomoses at the borders (Fig. 1). The central abdomen (zone I) is supplied by the superior epigastric arteries, and the inferior abdomen (zone II) by the deep and superficial inferior epigastric arteries, the deep and superficial circumflex iliac arteries, and the superficial external pudendal artery. Finally, the intercostal, subcostal, and lumbar segmental arteries supply the peripheral abdomen (zone III) [12]. The caudal excursion of an abdominal adipocutaneous flap

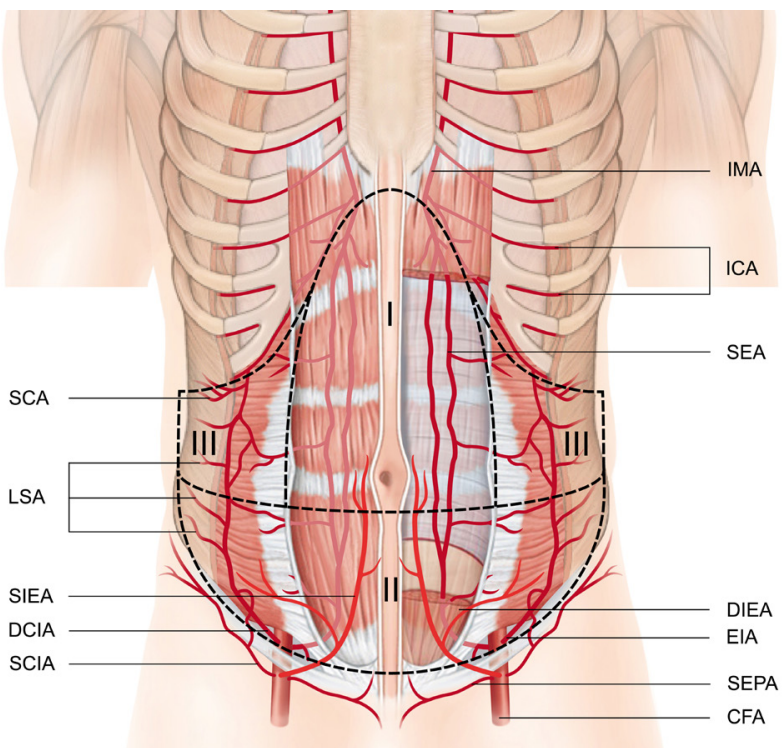

Fig. 1. Illustration representing abdominal wall vascularization. A scheme representing abdominal wall vascularization and anastomoses between vascular territories. The venous network is not shown for purposes of clarity. CFA, circumflex femoral artery; DCIA, indicates deep circumflex iliac artery; DIEA, deep inferior epigastric artery; EIA, external iliac artery; ICA, intercostal arteries; IMA, internal mammary artery; LSA, lumbar segmental arteries; SCA, subcostal arteries; SCIA, superficial circumflex iliac artery; SEA, superior epigastric artery; SEPA, superficial external pudendal artery; SIEA, superficial inferior epigastric artery.

(zone II), similar to what is performed in an abdominoplasty procedure, guarantees groin coverage in a simple and fast way. The abdominal adipocutaneous advancement flap has a reliable vascular supply based on anastomoses among these three areas. The abdominal adipocutaneous flap technique sacrifices the inferior blood supply and therefore indirectly relies on the superior epigastric arteries and the intercostal, subcostal, and lumbar segmental arteries.

Although previous reports have highlighted the reliability of abdominal adipocutaneous advancement flaps to reconstruct inguinal defects after oncologic resection, this has been rarely tested in case of complex groin defects after vascular surgery or in cases with infected materials and pressure sores, when standard techniques are unamenable $[13,14]$. The aim of this report was to evaluate the adipocutaneous abdominal flap as a salvage option if the usual groin reconstruction workhorse flaps are unavailable.

\section{METHODS}

\section{Study design}

We conducted a single-institution retrospective review. All patients admitted to the plastic surgery unit of a tertiary referral 
hospital between August 2015 and March 2019 who presented with groin defects were evaluated. In total, 15 patients were included. The first inclusion criterion was the presence of a chronic wound requiring debridement and soft tissue coverage by a flap (e.g., it was impossible to perform skin grafting due to threedimensional defects, vessels, or graft exposure). The second main criterion was a contraindication to perform a standard flap reconstruction, due to a compromised circumflex femoral artery network ( $n=9)$, lymphedema $(n=2)$ or scarring of the flap harvesting site $(n=3)$. A compromised circumflex femoral artery network was assessed by preoperative Doppler ultrasonography (e.g., inability to visualize the deep femoral artery or to establish flow) or after discussion with the consultant vascular surgeon who had performed the previous operation(s). A final patient presented relative contraindications for a standard flap reconstruction procedure (wasting syndrome in an elderly patient), while still needing a simple soft tissue coverage technique.

The patients presented with complex inguinal defects, as complications following vascular surgery with $(n=7)$ or without $(\mathrm{n}=3)$ prosthetic material (5 Omniflow II vascular prosthesis, Bio Nova International Pty Ltd., North Melbourne, Australia; 1 Gore Propaten Vascular Graft, W. L. Gore and Associates Inc., Santa Clara, CA, USA; 2 Silver Graft, Aesculap AG, Tuttlingen,
Germany), prosthetic material infection following general surgery $(\mathrm{n}=4)$ (1 DynaMesh-PP light, NBC Meshtec Inc., Tokyo, Japan; 1 Lubinus SPII, Waldemar LINK GmbH \& Co. KG, Norderstedt, Germany; 1 Versafit CC trio, Medacta International, Castel San Pietro, Switzerland; 1 Natural Knee prosthesis, Zimmer Biomet, Warsaw, Poland) and soft tissue necrosis following trauma $(\mathrm{n}=1)$ (Table 1$)$.

All patients with peripheral artery disease (PAD) were classified according to the Rutherford classification [15]. Patients' demographic characteristics and comorbidities are listed in Table $1[16,17]$. Informed consent was obtained from all patients, including approval for photographic documentation. The study protocol followed the ethical guidelines of the Declaration of Helsinki (1975).

\section{Surgical technique}

Under general anesthesia, all patients underwent extensive debridement and microbiological sampling. An adipocutaneous flap was raised over the abdominal fascia in a way similar to an ordinary abdominoplasty procedure (Fig. 2). A deep fat layer under the superficialis fascia was left intact over the external oblique muscles when possible, so as to preserve the lymphatic drainage and prevent seroma [18]. Dissection was pursued in a

Table 1. Demographic characteristics

\begin{tabular}{|c|c|c|c|c|c|c|}
\hline Patient & $\begin{array}{c}\text { Sex/age } \\
(y r)\end{array}$ & Etiology of inguinal defect & Comorbidities & $\begin{array}{l}\text { Contraindication to } \\
\text { standard reconstruction }\end{array}$ & $\begin{array}{l}\text { NPWT days } \\
\text { prior to flap surgery } \\
\text { (day) }\end{array}$ & $\begin{aligned} \mathrm{BMl} \\
\left(\mathrm{kg} / \mathrm{m}^{2}\right)\end{aligned}$ \\
\hline 1 & $\mathrm{~F} / 74$ & $\begin{array}{l}\text { latrogenic femoral arterio-venous fistula after } \\
\text { coronarography }\end{array}$ & CKD, DM, HL, HTN, IC, RC & Lymphedema & 9 & 40.5 \\
\hline 2 & $M / 69$ & ECMO for cardiac failure (LVEF <35\%) & CKD & Compromised CFA network & 40 & 29 \\
\hline 3 & $M / 82$ & Femoro-popliteal bypass for Rutherford 5 PAD & Active smoking, CKD, HL, HTN & Compromised CFA network & 16 & 29.3 \\
\hline 4 & $M / 64$ & Ilio-femoral bypass for Rutherford 5 PAD & Active smoking, DM, HL, HTN & Compromised CFA network & 43 & 28.7 \\
\hline 5 & $\mathrm{~F} / 55$ & $\begin{array}{l}\text { Infected hip prosthesis after hip replacement } \\
\text { for osteoarthritis }\end{array}$ & - & $\begin{array}{l}\text { Scarring of potential flap } \\
\text { harvest site }\end{array}$ & 30 & 55.9 \\
\hline 6 & $F / 84$ & $\begin{array}{l}\text { Vicryl mesh infection after reconstruction of an } \\
\text { evisceration }\end{array}$ & $\begin{array}{l}\text { Previous thoracotomy for lumbar } \\
\text { (L1) fracture osteosynthesis, HL }\end{array}$ & Lymphedema & 27 & 23.1 \\
\hline 7 & $83 / \mathrm{M}$ & Femoral pseudoaneurysm repair & $\begin{array}{l}\text { HTN, previously operated PAD } \\
\text { Rutherford } 5\end{array}$ & Compromised CFA network & 13 & 28.9 \\
\hline 8 & $F / 65$ & $\begin{array}{l}\text { Aorto-bifemoral bypass after abdominal aorta } \\
\text { aneurysm repair }\end{array}$ & Active smoking, CKD, HL, HTN & Compromised CFA network & 13 & 27.4 \\
\hline 9 & $M / 67$ & Femoro-popliteal bypass for Rutherford 5 PAD & $\begin{array}{l}\text { Cardiac failure (LVEF <35\%), DM, } \\
\text { CKD, HL, HTN }\end{array}$ & Compromised CFA network & 23 & 37.2 \\
\hline 10 & $F / 81$ & Hip prosthesis for osteoarthritis & DM, HTN & Scarring of harvest site & 12 & 37.9 \\
\hline 11 & $F / 86$ & $\begin{array}{l}\text { Posttraumatic anterior superior iliac spine } \\
\text { pressure sore }\end{array}$ & AF, HTN & Poor overall condition & - & 15.6 \\
\hline 12 & $M / 74$ & Femoro-popliteal bypass for Rutherford 5 PAD & Active smoking, HL, HTN & Compromised CFA network & - & 34 \\
\hline 13 & $M / 62$ & Necrotizing fasciitis after knee prosthesis & $\begin{array}{l}\text { Cardiac failure (LVEF }<35 \%) \\
\text { HTN, CRF }\end{array}$ & Scarring of harvest site & 34 & 37 \\
\hline 14 & $\mathrm{~F} / 58$ & Aorto-bifemoral bypass for Rutherford 3 PAD & Active smoking, HL, HTN & Compromised CFA network & 120 & 25 \\
\hline 15 & $\mathrm{~F} / 67$ & Ilio-femoral bypass for Rutherford 6 PAD & Active smoking, DM, CKD, HL, HTN & Compromised CFA network & 10 & 32 \\
\hline
\end{tabular}

NPWT, negative-pressure wound therapy; BMI, body mass index; F, female; M, male; ECMO, extracorporeal membrane oxygenation; LVEF, left ventricular ejection fraction; PAD, peripheral arterial disease; CKD, chronic kidney disease; DM, diabetes mellitus; HL, hyperlipidemia; HTN, arterial hypertension; IC, ischemic cardiopathy; RC, rhythmic cardiopathy; AF, atrial fibrillation; CRF, chronic renal failure; CFA, circumflex femoral artery. 

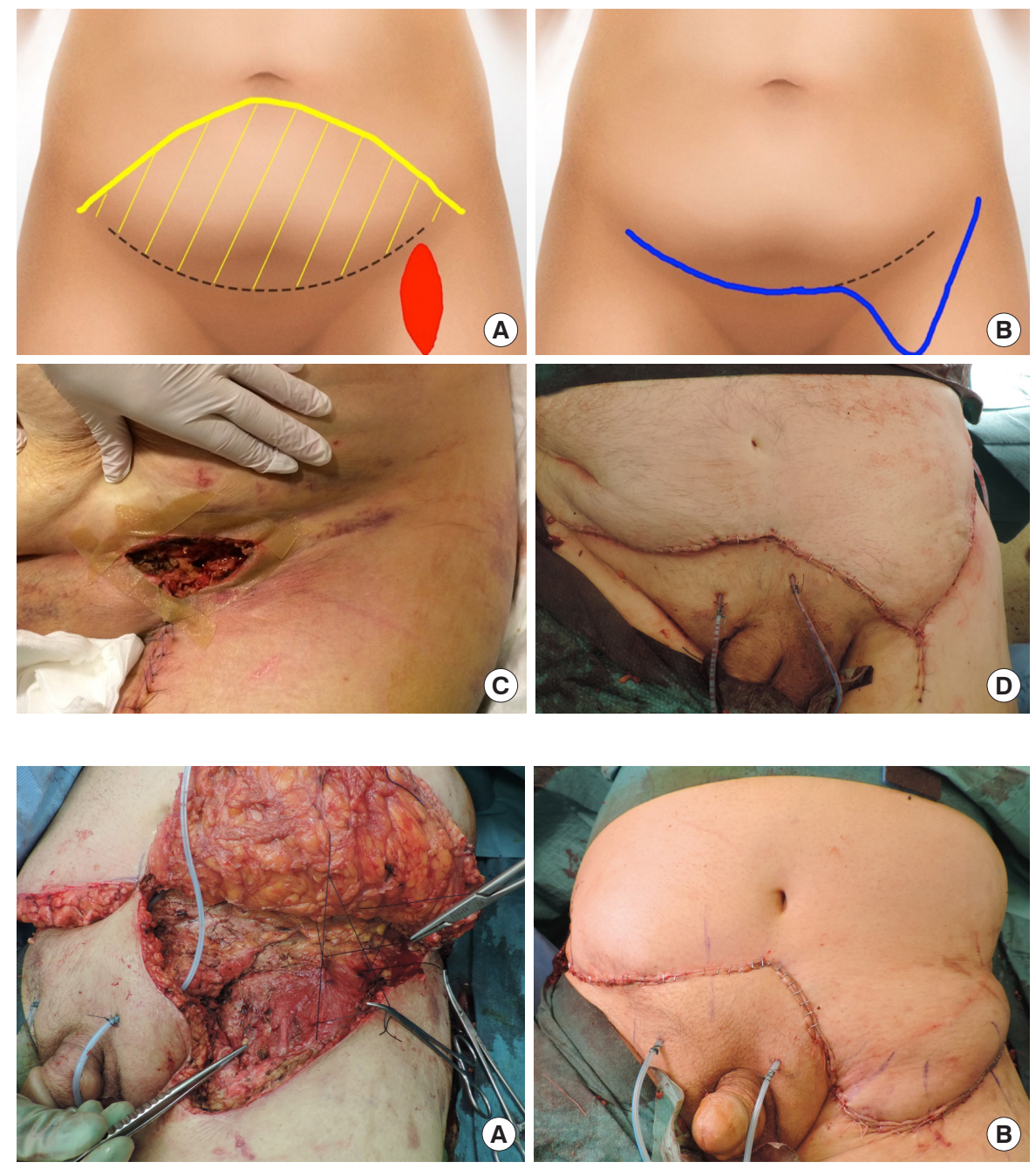

Fig. 2. Illustrations and photographs showing abdominal flap design. (A) The flap was designed from the left to the right anterior superior iliac spine (dashed line). The undermining (yellow area) extended below the umbilicus (yellow line), in order to cover the defect (red area). (B) Coverage of the defect was complete after closure (blue line). (C) Vascular surgical site exposure following unsuccessful vacuum therapy required debridement and coverage with an abdominal advancement flap. (D) A photograph showing the immediate postoperative result.

Fig. 3. Intraoperative and early postoperative pictures. (A) A photograph showing the exposed femoral vessels after debridement, the raised flap, and quilting sutures. (B) A photograph showing early postoperative defect closure. classical abdominoplasty fashion, without umbilical transposition, as allowed by abdominal laxity. After bilateral undermining, the adipocutaneous excess was tailored to provide effective coverage of the defect, while it was resected on the contralateral side. The distal edge of the flap was cut until sufficient arterial bleeding was achieved. If needed, the distal flap could be de-epithelized and fixed caudally to avoid shearing forces and improve stability [19]. In patients with preoperative lymphatic leakage and significant lymphorrhea, patent blue dye was injected in the dermis in the intermetatarsal space and along the great saphenous vein pathway to locate lymphatic leaks, which were sealed by sutures (Prolene 4.0, Ethicon Inc., Somerville, NJ, USA). After hemostasis and placement of two BLAKE drains (Ethicon Inc.), quilting sutures (Vicryl 1 and 2.0, Ethicon Inc.) were used to reduce tension on the skin edges and to minimize dead space (Fig. 3). The drains were removed with an output of less than $40 \mathrm{~mL}$ in 24 hours and after initiation of mobilization. Postoperatively, antibiotic treatment was adapted to antibiotic resistance testing, based on intraoperative microbiological samples.
Intravenous antibiotics were shifted to oral antibiotics after wound healing for a total of 2 weeks, or were prolonged for 3 months in cases of chronic graft infection [20].

\section{Postoperative management}

The flaps were monitored four times a day during the first 48 hours regarding color, temperature, and capillary refill, with particular attention to venous congestion. Subsequently, checks were performed twice daily. During the first 5-7 postoperative days (PODs), we recommended strict bed rest, allowing $30^{\circ}$ flexion of the hip. Starting in the second postoperative week, patients initiated mobilization with a physiotherapist.

Postoperative complications were systematically recorded and graded according to the previous literature (Tables 2, 3). Major flap-related complications included partial or total full-thickness flap necrosis (due to venous congestion or arterial insufficiency) and major wound dehiscence (more than one-third of the incision length) requiring surgical re-intervention. Minor complications encompassed minor wound dehiscence (less than one- 

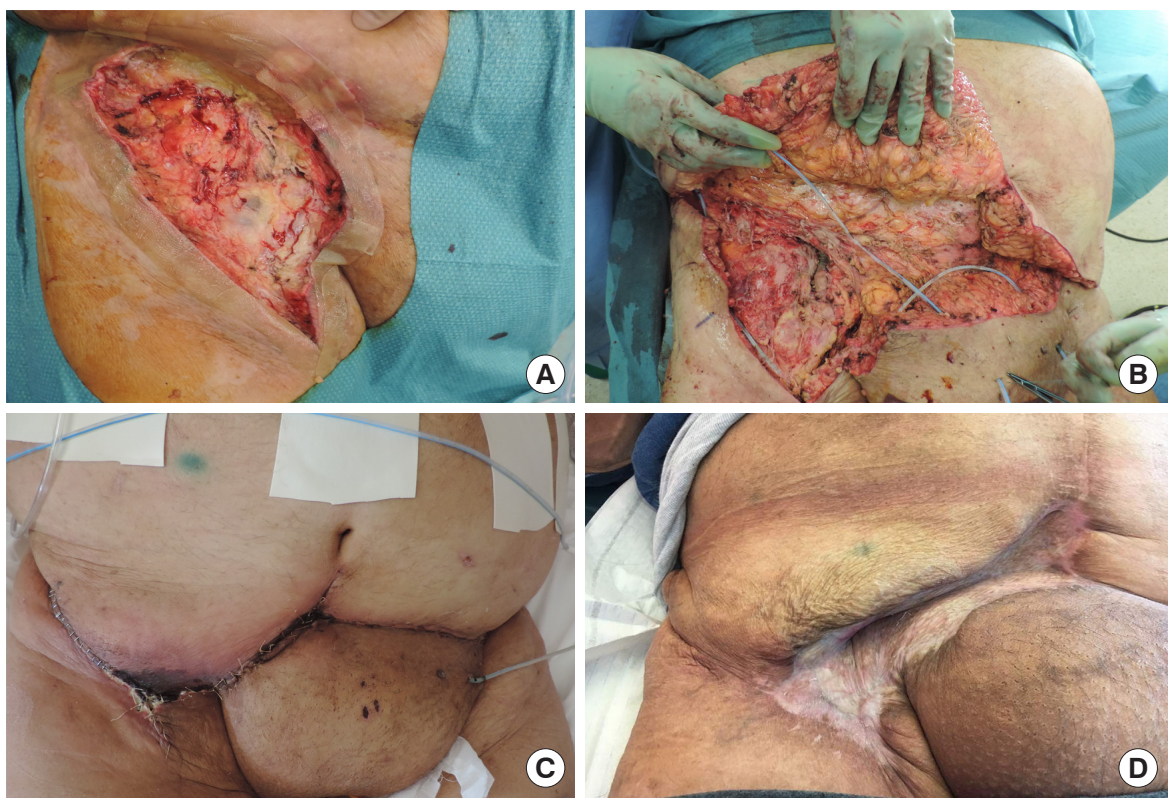

Fig. 4. Abdominal flap coverage with complications and management. (A) A photograph showing a right inguinal defect before debridement, following unsuccessful vacuum therapy. (B) Further debridement exposing the vascular synthetic graft with atonic and hypoperfused tissues. (C) Postoperative distal flap necrosis. (D) Despite flap debridement, the synthetic vascular graft was covered by sufficient tissue to allow a split-thickness skin graft, and provided effective coverage of the underlying structures at a 3-month follow-up.

Table 2. Clinical outcomes of the patients

\begin{tabular}{|c|c|c|c|c|c|c|c|c|}
\hline Patient & $\begin{array}{l}\text { Size of inguinal } \\
\text { defect }\left(\mathrm{cm}^{2}\right) \\
\text { (width } \times \text { length) }\end{array}$ & $\begin{array}{l}\text { Operative } \\
\text { time } \\
\text { (min) }\end{array}$ & $\begin{array}{l}\text { Drainage } \\
\text { time } \\
\text { (day) }\end{array}$ & Flap outcome & $\begin{array}{l}\text { Non-flap-related } \\
\text { complications }\end{array}$ & $\begin{array}{l}\text { Time to } \\
\text { healing } \\
\text { (day) }\end{array}$ & $\begin{array}{l}\text { Hospital } \\
\text { stay } \\
\text { (day) }\end{array}$ & $\begin{array}{l}\text { Follow-up } \\
\text { (mon) }\end{array}$ \\
\hline 1 & $80(8 \times 10)$ & 121 & 14 & Favorable & - & 17 & 17 & 43 \\
\hline 2 & $168(12 \times 14)$ & 110 & 12 & $\begin{array}{l}\text { Venous congestion requiring } \\
\text { debridement and flap advancement }\end{array}$ & - & 14 & $83^{\text {a) }}$ & $3^{b)}$ \\
\hline 3 & $56(7 \times 8)$ & 115 & 25 & Favorable & - & 23 & 27 & $7^{\text {c) }}$ \\
\hline 4 & $80(8 \times 10)$ & 110 & 9 & Delayed wound healing & - & 40 & 20 & 44 \\
\hline 5 & $120(10 \times 12)$ & 60 & 13 & Favorable & - & 16 & 15 & 46 \\
\hline 6 & $48(6 \times 8)$ & 104 & 1 & Favorable & - & 20 & 14 & 46 \\
\hline 7 & $117(9 \times 13)$ & 173 & 16 & Favorable & Hematoma drained surgically & 20 & 57 & 38 \\
\hline 8 & $72(8 \times 9)$ & 70 & 10 & Favorable & - & 14 & 28 & 38 \\
\hline 9 & $192(12 \times 16)$ & 240 & 27 & $\begin{array}{l}\text { Distal flap necrosis }(<1 / 3) \text { due to } \\
\text { arterio-venous insufficiency } \\
\text { requiring debridement, flap } \\
\text { advancement and STSG }\end{array}$ & - & 89 & 131 & 38 \\
\hline 10 & $168(12 \times 14)$ & 77 & 11 & Favorable & - & 15 & 12 & 35 \\
\hline 11 & $60(6 \times 10)$ & 97 & 8 & Favorable & - & 15 & 21 & 50 \\
\hline 12 & $110(10 \times 11)$ & 108 & 12 & Favorable & - & 21 & 23 & 36 \\
\hline 13 & $300(15 \times 20)$ & 286 & 5 & Favorable & & 21 & 24 & 9 \\
\hline 14 & $60(6 \times 10)$ & 114 & 7 & Favorable & $\begin{array}{l}\text { Neuropathic pain (lateral } \\
\text { femoro-cutaneous nerve) }\end{array}$ & 10 & 9 & 6 \\
\hline 15 & $60(6 \times 10)$ & 122 & 16 & Favorable & - & 21 & 25 & 5 \\
\hline Mean & 113 & 127 & 12 & & & 24 & 34 & 30 \\
\hline SD & 70 & 62 & 7 & & & 19 & 34 & 18 \\
\hline Median & 95 & 112 & 12 & & & 17 & 24 & 37 \\
\hline
\end{tabular}

STSG, split-thickness skin graft; SD, standard deviation.

${ }^{a}$ Hospital stay including prolonged stay after complete wound healing due to non-surgical comorbidities; ${ }^{b}$ Cardiac-related death at 3 months postoperatively; ${ }^{\text {c }}$ Death due to non-surgical comorbidities at 7 months postoperatively.

third of the incision length) healed by secondary intention, as well as superficial flap necrosis treated by minimal debridement and skin graft or local flap advancement. Surgical site infection, delayed wound healing, recurrence of lymphatic leakage, hema- toma, and seroma were recorded when present.

\section{Statistical analysis}

Patients' characteristics such as age, body mass index, defect 
Table 3. Classification of complications

\begin{tabular}{lll}
\hline \multicolumn{1}{c}{ Minor complications } & \multicolumn{1}{c}{ Major complications } \\
\hline Flap-related & Delayed wound healing $(<1 / 3$ of incision length $)$ & $\begin{array}{r}\text { Full-thickness distal flap necrosis }(<1 / 3 \text { of the incision length) requiring debridement, } \\
\text { flap re-advancement, and a split-thickness skin graft Progressive venous congestion } \\
\text { requiring re-intervention }\end{array}$ \\
Non-flap-related & Hematoma requiring surgical drainage & \\
\hline
\end{tabular}

size, operative time, drainage duration, time to healing, length of hospital stay, and length of follow-up were statistically analyzed (GraphPad Prism 5.0; GraphPad Software, San Diego, CA, USA). For each patient, we calculated the mean, the standard deviation (SD), and the range. Unless otherwise noted, average values are presented as mean $\pm \mathrm{SD}$.

\section{RESULTS}

\section{Patients' characteristics}

The study population was in overall poor condition, as 12 of 15 patients (80\%) had hypertension, 10 of 15 (67\%) had contaminated or infected prosthetic material, seven of 15 (47\%) had $\mathrm{PAD}$, five of 15 (33\%) had diabetes, seven of 15 (47\%) had chronic renal failure, and six of 15 (40\%) were current smokers (Table 1). In addition, 13 of 15 patients (87\%) were overweight, exhibiting moderate to severe abdominal adipocutaneous excess. In 13 of the 15 patients, negative-pressure wound therapy (NPWT) was used as a bridge technique before referral to our department (average of NPWT days, 30; median of NPWT days, 23). The mean body mass index was $32.1 \pm 9.2 \mathrm{~kg} / \mathrm{m}^{2}$ and nine of 15 patients $(60 \%)$ had hypercholesterolemia, although 12 of 15 patients ( $80 \%)$ had a preoperative serum albumin level $<30 \mathrm{~g} / \mathrm{L}$. Details on the vascular procedures and PAD stages are summarized in Table 1.

\section{Flap characteristics and operative time}

The mean surface area of the defects after debridement was $113 \pm 69 \mathrm{~cm}^{2}$ (range, $48-300 \mathrm{~cm}^{2}$ ). The mean operative time was $127 \pm 62$ minutes (range, 60-286 minutes). The mean duration of drainage after the operation was $12 \pm 7$ days (range, 1-27 days) (Table 2).

\section{Outcomes and complications}

We evaluated long-term outcomes with a mean follow-up of $2.4 \pm 1.5$ years. Soft tissue coverage was effective in all cases. Patients $1,3,5,6,8,10,11,12,13,14$, and 15 healed uneventfully. Patient 4 experienced delayed wound healing due to a minor flap-related complication, but the wound healed with local wound care. Patient 7 developed a hematoma on POD 1, which was a flap-unrelated complication due to early therapeutic hepa- rinization. The hematoma required surgical drainage, but then healed uneventfully.

Patients 2 and 9 developed major flap-related complications. Patient 2 , an elderly patient with severe cachexia and overall very poor condition, developed progressive venous congestion due to non-compliance with the positioning guidelines, and thus required surgical intervention (debridement of devitalized tissues and flap advancement) on POD 29, allowing uneventful wound healing. The patient's hospital stay was prolonged due to cardiac and neurological comorbidities, ultimately leading to premature death at 3 months postoperatively. Patient 9 , who initially presented with the second most extensive inguinal defect (192 $\mathrm{cm}^{2}$ ) and severe comorbidities (preoperative left ventricle ejection fraction of $23 \%$, PAD, hypertension, diabetes mellitus, chronic kidney disease, preoperative serum albumin $<30 \mathrm{mg} / \mathrm{L}$, body mass index of $37 \mathrm{~kg} / \mathrm{m}^{2}$, chronic inguinal lymphatic leak), developed full-thickness distal flap necrosis (Fig. 4). This complication required further debridement, flap re-advancement, reobliteration of lymphatic leaks, and a split-thickness skin graft for coverage of the caudal part of the defect on POD 20.

The mean duration of wound drainage was $12 \pm 7$ days. All patients eventually fully recovered with a time to complete healing of $24 \pm 19$ days, considering the total healing time if a second surgical intervention was warranted during the same hospital stay.

The mean length of stay at the hospital was $34 \pm 34$ days. All patients showed favorable outcomes with no wound breakdown or recurrence at a 6-month follow-up, except for patient 2, as discussed above. Patient 3 died 7 months postoperatively due to massive ear, nose, and throat bleeding while on anticoagulation therapy.

\section{DISCUSSION}

The current literature on reconstructive procedures after the failure of conservative treatment of VSSI contains heterogeneous data [21]. LoGiudice et al. [7] recently reported an overall complication rate of $30 \%-40 \%$ after VSSI or oncologic procedures in the groin area, requiring extensive debridement and coverage with ALT or RA flaps. Schutzer et al. [22] observed a mortality rate of $22 \%$ within a year after VSSI coverage with a sartorius muscle flap. A recent literature review reported that 
groin defects after vascular surgery were covered with muscle flaps in $95 \%$ of cases. Complications included infection in 14\% of cases, seroma in $7.5 \%$, delayed healing or flap dehiscence in $40.8 \%$, and an overall re-intervention rate of $20 \%$. These findings are consistent with our results [1].

Our data include three cases of re-intervention (20\%), only two of which were due to flap-related complications (15\%), presenting as distal flap necrosis due to venous congestion and flap arterial insufficiency (Table 3). Thus, although a larger cohort would have enabled a statistical analysis with greater power, these findings essentially correspond to the recently reported re-intervention rate of $30 \%$ after coverage of a VSSI in the groin area with a sartorius muscle flap. No complications occurred in our series in patients who underwent reconstruction after orthopedic prosthetic material infection or trauma, which is most probably due to a lower prevalence of comorbidities and smaller defects (range, $48-168 \mathrm{~cm}^{2}$ ) than those of the vascular patients (range, 56-192 $\mathrm{cm}^{2}$ ). This aligns with the possible correlation between the size of the soft tissue defect and the risk of postoperative complications. Even though defects associated with VSSI are usually limited in size, the cases in our study involved moderate-sized defects after debridement [4]. The extensive intraoperative undermining required to raise the abdominoplasty-like flap, as well as the strict supine position observed for 5 days postoperatively, probably contributed to the long drainage time recorded ( $>10$ days).

All flaps in our cohort survived and the wounds healed within 40 days (mean, $25 \pm 6$ days; median, 17 days), except for one patient who healed within 89 days. When data from the latter patient were excluded from statistical analysis, the adjusted mean healing time ( $24 \pm 19$ days) approached the global median value of 17 days. In the recent literature, LoGiudice et al. [7] observed mean times to healing of 42 days and 60 days using ALT and RA flaps, respectively, to cover groin defects following VSSI or oncologic procedures. Although our complication and re-intervention rates, along with the time to complete healing and the length of hospital stay, may appear high compared to other reports of flap surgery, the high prevalence of comorbidities among the patients in this study must be considered. In this cohort, $80 \%$ of the patients demonstrated a preoperative level of serum albumin $<30 \mathrm{~g} / \mathrm{L}$, which is a surrogate marker of their nutritional status and a confirmed risk factor for postoperative complications [17]. Even after complete healing, the hospital stay was often extended to allow full rehabilitation of these patients with multiple comorbidities before reintegration into society or transfer to supportive care facilities.

Importantly, although $73 \%$ of the patients included in this study presented exposed and contaminated synthetic vascular grafts, hip prostheses, or non-absorbable abdominal meshes, none developed postoperative wound breakdown fistulas or graft anastomosis leakage. This confirms the effectiveness of this particular flap coverage technique, with the synergistic effect of vascularized tissue and selective antibiotic therapy to control chronic wound infections. In patients suffering from severe PAD (47\%), predominantly affecting arteries in the lower limb, the use of an abdominal flap ensured reliable vascularization [23]. This method can be considered effective and reliable, with complete healing and satisfactory functional results achieved in all patients.

In this cohort, NPWT was mainly applied by other departments before referral, as a bridge therapy or for wound preconditioning. Prolonged NPWT garments may promote the presence of the chronic bacterial load, and thus the duration of the NPWT bridge therapy should be shortened in order to lower the risk of a secondary and polymicrobial wound infection [24]. As a general rule, in cases of non-successful NPWT therapy, we would suggest that flap coverage should not be delayed for over 2 weeks, especially in the presence of an exposed synthetic graft. In our experience, the main indications for adipocutaneous abdominal flaps in groin reconstruction are failure of conservative treatment in the setting of a compromised deep femoral artery network, which can jeopardize the vascularization of the usual flaps based on the circumflex femoral artery, and poor thigh tissue quality caused by scarring or edema in patients with severe comorbidities. This procedure could be performed under spinal anesthesia without discomfort, which is pertinent for patients with contraindications to general anesthesia. The main requirements for an effective reconstruction are sufficient abdominal laxity and radical debridement. However, previous open abdominal surgery, hernias, and bulky abdomens with wall instability may contraindicate this option. Non-cooperative patients may not benefit from this technique, since the above-mentioned postoperative positioning guidelines are essential to allow wound healing and prevent complications. Morbidly obese patients require careful evaluation, as an oversized flap may favor venous congestion. This technique could also potentially be applicable in cases of tumor-related wound healing disorders in the groin region (e.g., after urological resection or lymphadenectomy), extending its indications, even if its applicability in such scenarios would require further clinical evaluation.

The low absolute number of patients included in the study, due to the restricted target population, prevented us from fully elucidating the complications of this technique through a proper statistical analysis; nonetheless, the strict patient inclusion criteria, the analysis of a prospectively maintained database, and the homogeneous comorbidities of the patients enabled consistent results to be obtained. Although a direct comparison with 
workhorse thigh flaps is missing, this technique represents a surgical option when standard flaps pathways are not available, providing a rescue reconstructive procedure for groin wound coverage in a population prone to complications. Although this method is technically simple, experience and care are required when placing the flap; it should provide protection to vascular structures without tension to guarantee a vascular supply to hypoxic and poorly perfused tissues.

Abdominal adipocutaneous flaps may be a useful and simple solution for inguinal coverage, with reproducible outcomes, when applied to selected cases to cover moderate defects in the groin area.

\section{NOTES}

\section{Conflict of interest}

No potential conflict of interest relevant to this article was reported.

\section{Ethical approval}

The study was approved by the commission d'éthique du canton de Vaud (CER-VD) (approval No. ProjectID 2021-00963) and performed in accordance with the principles of the Declaration of Helsinki. Written informed consent was obtained.

\section{Patient consent}

The patients provided written informed consent for the publication and the use of their images.

\section{Author contribution}

Conceptualization: C Schaffer, D Haselbach, PG di Summa. Data curation: C Schaffer, D Haselbach, L Schiraldi. Formal analysis: C Schaffer. Methodology: C Schaffer, D Haselbach, PG di Summa. Writing - original draft: C Schaffer. Writing - review \& editing: C Schaffer, K Sörelius, DF Kalbermatten, W Raffoul, PG di Summa.

\section{ORCID}

$\begin{array}{ll}\text { Clara Schaffer } & \text { https://orcid.org/0000-0001-7230-4984 } \\ \text { Daniel Haselbach } & \text { https://orcid.org/0000-0001-5394-6527 } \\ \text { Luigi Schiraldi } & \text { https://orcid.org/0000-0002-9766-5185 } \\ \text { Karl Sörelius } & \text { https://orcid.org/0000-0001-7747-6339 } \\ \text { Daniel F. Kalbermatten }\end{array}$

https://orcid.org/0000-0002-6626-1233

Wassim Raffoul https://orcid.org/0000-0002-3524-0077

Pietro G. di Summa https://orcid.org/0000-0002-1431-4479

\section{REFERENCES}

1. Sorelius K, Schiraldi L, Giordano S, et al. Reconstructive surgery of inguinal defects: a systematic literature review of surgical etiology and reconstructive technique. In Vivo 2019;33:1-9.

2. Evans GR, Francel TJ, Manson PN. Vascular prosthetic complications: success of salvage with muscle-flap reconstruction. Plast Reconstr Surg 1993;91:1294-302.

3. Zelken JA, AlDeek NF, Hsu CC, et al. Algorithmic approach to lower abdominal, perineal, and groin reconstruction using anterolateral thigh flaps. Microsurgery 2016;36:104-14.

4. Fischer JP, Mirzabeigi MN, Sieber BA, et al. Outcome analysis of 244 consecutive flaps for managing complex groin wounds. J Plast Reconstr Aesthet Surg 2013;66:1396-404.

5. Celik N, Wei FC, Lin CH, et al. Technique and strategy in anterolateral thigh perforator flap surgery, based on an analysis of 15 complete and partial failures in 439 cases. Plast Reconstr Surg 2002;109:2211-6.

6. Chatterjee A, Kosowski T, Pyfer B, et al. A cost-utility analysis comparing the sartorius versus the rectus femoris flap in the treatment of the infected vascular groin graft wound. Plast Reconstr Surg 2015;135:1707-14.

7. LoGiudice JA, Haberman K, Sanger JR. The anterolateral thigh flap for groin and lower abdominal defects: a better alternative to the rectus abdominis flap. Plast Reconstr Surg 2014;133:162-8.

8. Alkon JD, Smith A, Losee JE, et al. Management of complex groin wounds: preferred use of the rectus femoris muscle flap. Plast Reconstr Surg 2005;115:776-83.

9. Scaglioni MF, Enrique Carrillo Jimenez L, Kuo YR, et al. Pedicled posteromedial thigh (PMT) flap: a new alternative for groin defect reconstruction. Microsurgery 2017;37:33943.

10. Morasch MD, Sam AD 2nd, Kibbe MR, et al. Early results with use of gracilis muscle flap coverage of infected groin wounds after vascular surgery.J Vasc Surg 2004;39:1277-83.

11. Mathes SJ, Nahai F. Classification of the vascular anatomy of muscles: experimental and clinical correlation. Plast Reconstr Surg 1981;67:177-87.

12. Johnson TG, Von SJ, Hope WW. Clinical anatomy of the abdominal wall: hernia surgery. OA Anatomy 2014;2:3.

13. Tabatabaei S, McDougal WS. Primary skin closure of large groin defects after inguinal lymphadenectomy for penile cancer using an abdominal cutaneous advancement flap. J Urol 2003;169:118-20.

14. Sisti A, Tassinari J, Cuomo R, et al. A case of extramammary inguinal Paget disease in a male patient: surgical treatment 
with an abdominal advancement cutaneous flap. Acta Biomed 2017;88:79-81.

15. Rutherford RB, Baker JD, Ernst C, et al. Recommended standards for reports dealing with lower extremity ischemia: revised version. J Vasc Surg 1997;26:517-38.

16. Eagle KA, Berger PB, Calkins H, et al. ACC/AHA guideline update for perioperative cardiovascular evaluation for noncardiac surgery--executive summary: a report of the American College of Cardiology/American Heart Association Task Force on Practice Guidelines (Committee to Update the 1996 Guidelines on Perioperative Cardiovascular Evaluation for Noncardiac Surgery). J Am Coll Cardiol 2002;39: 542-53.

17. Weimann A, Braga M, Carli F, et al. ESPEN guideline: clinical nutrition in surgery. Clin Nutr 2017;36:623-50.

18. Le Louarn C, Pascal JF. High superior tension abdominoplasty. Aesthetic Plast Surg 2000;24:375-81.

19. Erba P, di Summa PG, Raffoul W, et al. Tip anchor flap in decubital surgery. Aesthetic Plast Surg 2011;35:1133-6.

20. Revest M, Camou F, Senneville E, et al. Medical treatment of prosthetic vascular graft infections: review of the literature and proposals of a Working Group. Int J Antimicrob Agents 2015;46:254-65.

21. Seify H, Moyer HR, Jones GE, et al. The role of muscle flaps in wound salvage after vascular graft infections: the Emory experience. Plast Reconstr Surg 2006;117:1325-33.

22. Schutzer R, Hingorani A, Ascher E, et al. Early transposition of the sartorius muscle for exposed patent infrainguinal bypass grafts. Vasc Endovascular Surg 2005;39:159-62.

23. Ouriel K. Peripheral arterial disease. Lancet 2001;358:125764.

24. Chan M, Yusuf E, Giulieri S, et al. A retrospective study of deep sternal wound infections: clinical and microbiological characteristics, treatment, and risk factors for complications. Diagn Microbiol Infect Dis 2016;84:261-5. 